\title{
Fuzzy evaluation of risk for transmission lines on-line monitoring
}

\author{
Wei Song, Haibing Zhang \\ State Grid Chongqing Electric Power Company, China
}

\begin{abstract}
Now many on-line monitoring systems of the transmission are relatively in a single mode. But the transmission lines are often in a more complex environment. A single monitoring cannot meet the requirements of safe, economic and efficient operation of transmission lines. So this paper proposes a fuzzy evaluation of risk on transmission lines based on on-line monitoring data. Firstly, this paper builds the hierarchy model of evaluation from the risk of transmission lines on probability attribute. Then the various risks that exist in the trans-mission lines can be effectively analyzed. Finally, the numerical example results show the effectiveness of the algorithm.
\end{abstract}

KEYWORD: Transmission Line; fuzzy evaluation; on-line monitoring; evaluation of risk

\section{INTRODUCTION}

Transmission lines are an important part of power system. It is wider coverage than substations. It is vast area in China. The transmission line through mountains, basin, hills and the terrain, along the transmission line corridors, and the geological condition is complex, climate changeable. There are often strong wind, thunder, heavy pollution, extreme weather conditions, such as snow and ice. Exposed to the environment, transmission line running status directly affected by the environment. Since the last century, many areas in China, which have both had suffered weather disasters caused by the transmission line fault. Transmission lines in the event of failure will directly affect the safe and stable operation of power system. It can bring great losses to the production and living of the national economy and harm to the national economic development and social stability.

Intelligent features of monitoring system for transmission lines is able to achieve transmission line equipment state diagnosis, evaluate the equipment running status and decision making. Monitoring system to provide data include: line running electric parameters, monitoring and meteorological environment parameters, equipment location, maintenance records, etc. At present various monitoring systems are independent of each other, monitoring data was not linked with transmission line components Nigol, O \& Buchan P.G. (1981), Yu P. (1992), Xiaoming Rui. (2011), Bjerkan Leif. (2009). While line fails, the answer is straight is a circuit el- ement. So it is necessary to analyze the monitoring system for intelligent decision-making system and realize the use of the existing monitoring data. Through the analysis, comparison, judgment, finally realizes to evaluate the running state of the line. Through the analysis of mass data processing, potential fault diagnosis in time, on the line to run before the hidden trouble in troubleshooting in a timely manner.

This paper builds the hierarchy model of evaluation from the risk of transmission lines on probability attribute using fuzzy evaluation theory. The various risks that exist in the transmission lines are effectively analyzed.

\section{TRANSMISSION LINE RISK COMPREHENSIVE EVALUATION BASED ON ON-LINE MONITORING DATA}

\subsection{All levels evaluation model}

State evaluation index system including evaluation eight indexes: basic state evaluation, tower state evaluation and guide ground state evaluation, evaluation of insulator state evaluation, overhead lines, hardware status evaluation, grounding device status evaluation and ancillary facilities state evaluation 8 indicators.

Ancillary facilities of the above indicators, such as state evaluation index on statistics has certain technical difficulty and omissions, inevitable existence description information is incomplete, fuzzy in- 
formation, and so on and so forth, this creates for each index and risk factors for the difficulty of mathematical quantification. For this kind of evaluation system with fuzzy factors, need to analytic hierarchy process (AHP) and fuzzy comprehensive evaluation combined two methods for processing Zhewen Jiang.(2004), Shuli Hu.(1994), Jie Zhang.\& Xinjing Chao.(2010). The rest can be produced in recent years, the frequency of the quantitative indi- cators, security implications can be obtained by online monitoring system for quantitative data.

State evaluation index system of hierarchy. The first layer for transmission line state evaluation target. The second rule of risk probability and risk effect as layer. The third layer above eight specific indicators as plan. Using the AHP method to determine the weight of each level and the overall weight. The layer is shown in Fig.1.

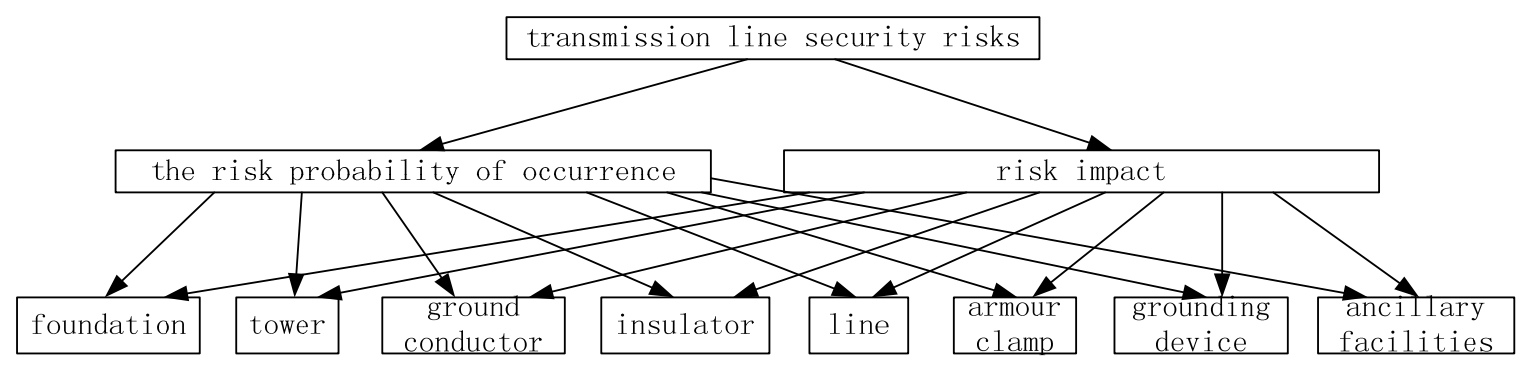

Figure 1. Indicators of risk structure model class time

\subsection{The establishment of the evaluation mode}

The evaluation index system of the single index evaluation calculation, the results into a membership function to calculate the single index, calculation under the measure of the membership value. Further evaluation index set. Then you can have to risk assessment value. The risk assessment value $S$ is calculated by

\section{$S=W_{\mathrm{s}} * W$}

Where $W_{s}, W$ is weight matrix and measure, $*$ is fuzzy operator.

\subsection{Transmission line comprehensive fuzzy evaluation based on on-line monitoring data}

To determine the various indicators of risk, this paper uses the method of fuzzy mathematics. Using the probability and impact of transmission line equipment disaster size grade, describe the risk of evaluation index values. It can describe a very simple and effective method for evaluating the risk indicators. Therefore, this paper adopts this approach to indica- tors of risk assessment and risk indexes of frequency and influence size measurement level.

The risk probability levels are ignored, very low, low, middle degree, high, very high, extra high. So the correspond frequency are not occur in general, 2-3 times/5 years, 1 time/ 1 year, 1 time/6 month, 1 times/1 month, many times/1 month, many times/1 day. All above data is get by on-line monitoring data.

The influence of transmission line risk occurs are best, better, normal, bad, very bad. So the correspond influence to power systems are no impact to overall system security, a small part system caused by a fault occurs, one part of the system caused by failure, serious cause system failure, cause the system paralysis.

Based on reference Shuli Hu.(1994), risk frequency fuzzy comprehensive evaluation results based on monitoring data are shown in Table.1.

Based on reference Shuli Hu.(1994), risk affect the fuzzy comprehensive evaluation based on monitoring data are shown in Table.2.

Table.1 Risk frequency fuzzy comprehensive evaluation result table

\begin{tabular}{|l|l|l|l|l|l|c|c|}
\hline Sub factors & Ignored & Very low & low & middle degree & high & very high & extra high \\
\hline Foundation & 46 & 14 & 11 & 8 & 7 & 4 & 0 \\
\hline Tower & 36 & 49 & 8 & 0 & 5 & 6 & 0 \\
\hline Ground Conductor & 42 & 21 & 13 & 35 & 8 & 0 & 0 \\
\hline Insulator & 33 & 22 & 24 & 8 & 4 & 13 & 0 \\
\hline Line & 32 & 38 & 17 & 7 & 0 & 0 & 0 \\
\hline Amour Clamp & 40 & 28 & 17 & 5 & 3 & 4 & 2 \\
\hline Ground device & 14 & 33 & 12 & 0 & 6 & 0 & 7 \\
\hline Ancillary facilities & 27 & 9 & 29 & 0 & 0 \\
\hline
\end{tabular}


Table.2 Risk affect fuzzy comprehensive evaluation result table

\begin{tabular}{|l|l|l|l|l|l|}
\hline sub-factors & best & better & normal & bad & very bad \\
\hline foundation & 13 & 29 & 9 & 0 & 0 \\
\hline tower & 41 & 37 & 21 & 7 & 0 \\
\hline Ground conductor & 25 & 45 & 23 & 11 & 3 \\
\hline insulator & 46 & 23 & 34 & 19 & 0 \\
\hline line & 61 & 11 & 21 & 6 & 3 \\
\hline amour clamp & 37 & 19 & 47 & 0 & 5 \\
\hline ground device & 29 & 21 & 51 & 0 & 7 \\
\hline ancillary facilities & 19 & 22 & 48 & 5 & 0 \\
\hline
\end{tabular}

$0.25,0.75,3,5.25,2$, 3. Programming with c lan-

\section{TRANSMISSION LINE STATE EVALUATION} RESULTS

According to the statistical data of a certain transmission line on-line monitoring, record is as follows: risk factors in the form of the statistical time frequency average of four years are as follows: 1.5, 23, 11, 13, guage can be very easy to implement risk assessment algorithm. Because of evaluation process and record data exist uncertainty. So using the method of using the literature Zhewen Jiang.(2004) in calculating effectiveness. The resulting risk factors assessment results as shown in Table. 3. F1-F7 is indicators of risk frequency level in Table.3.

Table.3 Transmission line risk assessment data

\begin{tabular}{|l|l|l|l|l|l|l|l|l|}
\hline sub-factors & F1 & F2 & F3 & F4 & F5 & F6 & F7 & validity of the measure \\
\hline foundation & 0 & 0.4 & 0.5 & 0 & 0 & 0 & 0 & 0.65 \\
\hline tower & 0.23 & 0 & 0 & 0 & 0 & 0.89 & 0.02 & 0.954 \\
\hline Ground conductor & 0 & 0.03 & 0 & 0 & 0.86 & 0.093 & 0 & 0.345 \\
\hline insulator & 0 & 0 & 0 & 0.56 & 0.32 & 0.95 & 0.05 & 0.886 \\
\hline line & 1 & 0 & 0 & 0.16 & 0 & 0 & 0 & 1 \\
\hline amour clamp & 0.6 & 0.4 & 0.8 & 0 & 0 & 0 & 0 & 0.649 \\
\hline ground device & 0.76 & 0 & 0.6 & 0.4 & 0 & 0 & 0 & 0.645 \\
\hline ancillary facilities & 0 & 0.66 & 0 & 1 & 0 & 0 & 0 & 1 \\
\hline
\end{tabular}

It can very clearly see that the ground, the three poles, insulator the highest probability of occurrence of a risk. So in the daily operation of the transmission line, we should strengthen the security of the system in these areas to ensure the safe and stable operation of the whole system.

Each risk index comprehensive evaluation importance as shown in Table. 4. V1-V5 is indicators of risk level.

Table.4 Risk index devaluation importance

\begin{tabular}{|l|l|l|l|l|l|l|}
\hline sub-factors & V1 & V2 & V3 & V4 & V5 & validity of the measure \\
\hline foundation & 0 & 0.5 & 0.7 & 0 & 0 & 0.524 \\
\hline tower & 0 & 0.2 & 0.6 & 0 & 0.3 & 0.411 \\
\hline Ground conductor & 0.3 & 0.8 & 0.1 & 0.2 & 0 & 0.375 \\
\hline insulator & 0 & 0.7 & 0.3 & 0 & 0 & 0.536 \\
\hline line & 0 & 0 & 0 & 0.2 & 0.2 & 1 \\
\hline amour clamp & 0 & 0.4 & 0 & 0 & 0 & 0.649 \\
\hline ground device & 0 & 0 & 0 & 0 & 0 & 0.479 \\
\hline ancillary facilities & 0 & 0.66 & 0 & 0.2 & 0 & 0.529 \\
\hline
\end{tabular}

It could be seen very clearly with the insulator, ancillary facilities, and the three kinds of risks for higher risk. In the daily operation of the transmission line, therefore, should strengthen the security of the system in these areas, to ensure the safe operation of the transmission line assessment is implementation. It provides maintenance decisionmaking basis for operation maintenance and management. The safeguard measures are completed in advance and eliminate the line potential hazard.

\section{CONCLUSIONS}

By using the transmission line theory of fuzzy evaluation, depth mining monitoring data, the analysis, comparison of monitoring data, identification of monitoring data, and risk evaluation of equipment state of transmission. The transmission line state risk

\section{REFERENCE}

Bjerkan Leif.2009. Application of fiber-optic bragg gratin sensors in monitoring environmental loads of overhead power transmission lines[J]. Applied Optics,2009,39(4): 554-560. 
Jie Zhang.\& Xinjing Chao.2010. Safety production capacity research Gui based on analytic hierarchy process. Light Industry Machine, 2010,15(2):18-23.

Nigol,O\& Buchan P.G.1981. Conductor Galloping Torsional Mechanism[J].IEEE Transactions on Power Apparatus and Systems, 1981,100(2): 708-720.

Shuli Hu.1994. Fuzzy sets and applications.Chengdu, Sichuan University Press,1994.

Xiaoming Rui.2011. On-line Monitoring System on Power Transmission Line Galloping Base on Fiber Grating Sensors[C]. Control Conference(CCC), 2011 30th Chinese, 2011: 4327-4330.

Yu P.1992. Inertial Coupled Galloping of Iced Conductors[J]. Journal of Applied Mechanics-transactions of the ASME, 1992,59(1): 140-145.

Zhewen Jiang.2004. Fuzzy mathematics tutorial. Beijing: National Defence Industry Press,2004 